\section{The Colouring of Maps}

THE countries of a map are sometimes given colours in such a way that no two countries which have a common frontier line are coloured the same. Mapmakers have long known empirically that four colours are sufficient to colour a map in this way, provided they are distributed correctly. In the nineteenth century, de Morgan and Cayley directed attention to this, and unsuccessful attempts were made to prove or disprove that any map drawn on the surface of a sphere or on a plane can be coloured, using at most four colours. This question, known as the four-colour problem, is to-day one of the most famous unsolved problems in mathematics. Heawood ${ }^{1}$ has proved that five colours are always sufficient.

When the four-colour problem could not be solved, the colouring of maps drawn on more complicated surfaces than the sphere was considered. Heawood ${ }^{2}$ proved that for all $h>1$, any map drawn on any surface of connectivity $h$ can always be coloured with, at most, $n_{h}$ colours, where

$$
n_{h}=\left[3 \frac{1}{2}+\frac{1}{2} \sqrt{ }(24 h-23)\right] .
$$

( $[x]$ denotes the greatest integer not exceeding $x$. For the sphere and the plane $h=1$, and substituting $h=1$ gives $n_{1}=4$.) For $h=2,3,4,5,7,9,11,13$, 15 , it is known that Heawood's number is the best possible ${ }^{2}$, in the sense that for these values of $h$ there are surfaces of connectivity $h$ on which maps requiring $n_{h}$ colours to colour them can be drawn. For all other values of $h$ greater than 1, $n_{h}$ is an upper bound for the required number $k_{h}$, the number such that there exists a surface of connectivity $h$ on which a map requiring $k_{h}$ colours to colour it can be drawn; but there exists no surface of connectivity $h$ on which a map requiring more than $k_{h}$ colours can be drawn. The determination of $k_{h}$ may be called the mapcolouring problem for surfaces of connectivity $h$.

I have recently been able to improve on this situation. It is convenient to call a map 'critical', if deleting any arbitrarily chosen country from it decreases the number of colours needed for its colouring. Clearly, any map is either critical, or a critical map which needs the same number of colours may be obtained from it by deleting suitably chosen countries. In two forthcoming papers ${ }^{3}$, I have proved the following :

(1) For all $h>1$, a map needing $n_{h}$ colours to colour it can be drawn on a surface of connectivity $h$ if, and only if, a map consisting of $n_{h}$ mutually adjacent countries can be drawn on the surface.

(2) For $8 \leqslant k \leqslant n_{h}$ a critical map drawn on a surface of connectivity $h$, which needs $k$ colours to colour it, contains at most $6(h-3) /(k-7)$ countries.

(1) can be used to decide whether $k_{h}=n_{h}$ or $k_{h}<n_{h}$ if $h>1$. It is only necessary to consider the different types of surfaces of connectivity $h$ and to verify whether a map consisting of $n_{h}$ mutually adjacent countries can be drawn on one of them. (2) enables one to determine $k_{h}$ in a finite number of steps if $h>1$, because there is only a finite number of essentially different maps containing not more than $6(h-3)$ countries.

It follows that, for all $h>1, k_{h}$ can be determined in a finite number of steps. In a sense, therefore, the problem of map colouring for surfaces of connectivity greater than 1 may be considered solved, and there remains only the four-colour problem. It is this circumstance, that the case $h=1$ is still unsolved, while all the other, at first sight much more complicated, cases can now be settled, which makes the four-colour problem so very remarkable and interesting.

\section{King's College,}

London, W.C.2.

Dec. 1.

1 Heawood, P. J., Quart. J. Math., 24, 332 (1890).

"Ibid. Also Hilbert and Cohn-Vossen, "Anschaulische Geometrie", 297. ${ }^{3}$ Canad. J. Math., and J. London Math. Soc., respectively.

\section{A Totally Sex-linked Gene in the House Mouse}

The first clearly proved case of total sex-linkage in the house mouse has been found - a fact worthy of record since very few sex-linked genes are yet known in mammals other than man, and none in the rodents. Apart from man, where hæmophilia and colour-blindness are the best known of a number of more or less certainly sex-linked genes ${ }^{1}$, there are only yellow in cats ${ }^{2}$ and hæmophilia in dogs ${ }^{3}$. In the mouse two genes, mottled and brindled, have been described $^{4}$ which may be totally sex-linked; but this cannot be directly proved as both genes are lethal in males. A test using females heterozygous for the new gene and for mottled or brindled should settle this point.

The new gene is semi-dominant and behaves in every way as would be expected of a totally sexlinked gene with no homologue on the $Y$-chromosome, though the numbers of mice bred are not yet enough to detect minor deviations from expectation. There are three phenotypes in females,-corresponding to the three genotypic classes; but there are only two in males, mutant males resembling the homozygous mutant females. This provides prima facie evidence of the existence of a differential segment in the sex chromosome of the mouse. The

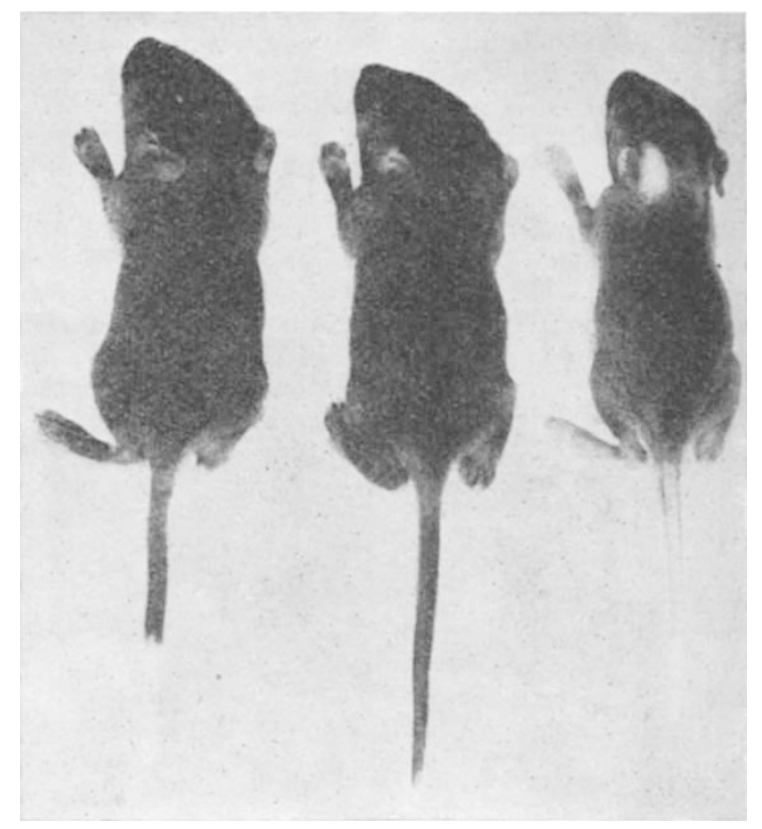

Phenotypic effects of the sex-linked gene $t a b b y$, illustrated in 12-day old mice. Left, normal $(++q$ or $+\delta)$; centre, heterozygous female $(T a+)$ right, homozygous female (TaTa) or hemizygous male $\left(T^{\prime} a\right)$ 